\title{
Application of cooling water supply systems thermography monitoring on the nuclear power plants
}

\author{
by M. Bazaleev, B. Banduryan, V. KLepikov, S. Donets, V. Lytvynenko, Ye. Prokhorenko, O.Startsev \\ Institute of Electrophysics and Radiation Technologies National Academy of Science of Ukraine, \\ Chernyshevsky St. 28, PO Box 8812,61002 Kharkiv, Ukraine, vvlytvynenko@ukr.net
}

\begin{abstract}
Application of cooling water supply systems thermography monitoring on the nuclear power plants was considered in this work. The main purpose was to improve the monitoring techniques of the technical-water supply control system on NPP, and to study the peculiarities of the heat occurrence happened under leakage formation on water level. The determination technique of painted coating peeling initial stage was developed. The temperature fields of spray ponds shields and pumping facility were measured and numerically analysed using fractal and segmentation methods. Possible mechanisms for the heat occurrence on a soil surface were discussed. The fractal dimensions fields of noises were built.
\end{abstract}

\section{Introduction}

Increasing of NPP safety requirements, caused by the accident on "Fukushima-1" NPP, expected to implement state monitoring methods of equipment, communications and water reserves. Their operability determines both beforethe-fact prevention of accidents occurrence and controllability for accident liquidation. Obviously, the variety of emergency and hazardous factors requires using the comprehensive approach to their monitoring based on the use of the modern techniques of measuring physical fields. One of these methods is the thermal imaging control. It is lately used for various technological equipment diagnostics. This paper presents the results of research, which goals were the development and improvement of infrared thermography control methods on the NPPs, particularly to monitor the cooling water supply systems.

In this work we conducted fractal analysis to characterize thermograms. We tried to understand new opportunities of such potential processing technique in increasing thermograms' informativeness. The fractal characteristics of thermograms are caused by two main factors [1-6]. First, the physical object of research is characterized by fractional dimension, therefore a thermal conductivity values differ from classical ones. Second, existing noise in the thermograms is also fractals. The concept of the fractal nature of thermographic data is still ambiguous, and requires a comprehensive exploration. It is also important to study a multifractal character of a thermal images sequence, because it may give us some information about the dynamics of temperature field.

\section{Materials and methods}

We carried out measurements using thermal imagers Fluke Ti32, Land Ti814, which of both has the temperature detection matrix resolution of $320 \times 240$, with the equal absolute accuracy at measuring temperature $0.05 \mathrm{~K}$. Thermal field distributions of soil surface, underground pipelines, pumping equipment, stop valves, facilities of spray ponds at NPPs were acquired (figure 1). Some measurements were carried out at night when temperature differences are bigger and the peculiarities of heat transfer are even more visible. Our measurements include: the detection of thermal field anomalies, leakages, corrosion of coatings, possible damage, and crack propagation in the equipment.

Using thermal imager Fluke Ti32 a sequence of three thermograms (table 1, figure 2-7) of homogeneous natural ground cover was recorded with time interval 15 min (IR $110-0 \mathrm{~min}$, IR 111 - $15 \mathrm{~min}$, IR $112-30 \mathrm{~min}$ ). The thermograms of soil surface (where underground utility lines are buried) were subjected to fractal analysis. In that case, the strongest type of self-similarity of fractals is not correct, so that we were dealing only with the statistical self-similarity. It is necessary to highlight, that the final result is even affected by the nature of the observed object: its heterogeneity, texture, stationary, shape, position in space, the projection on the detector's surface. Therefore the relative simplicity of the grass cover as the object of research is essential to prove correctness of obtained results, for further application of fractal analysis for more complex objects. The resulting measurement quality also depends on the thermal imager's absolute accuracy that takes into account changes of the technical parameters such as the supply, operating temperature, humidity etc. Free Java-based software ImageJ 1.47v and a plugin FracLac2014Aprb1012 [7] were used to calculate their fractal dimensions distributions. The way to define both the fractal and the multifractal dimensions of the thermograms was based on the box counting method [7, 8], and maximum relative error did not exceed $6 \%$.

In this work we carried out fractal dimension analysis of binary and gray-level images, all RGB figures shown here only for the sake of clarity. 
We built fractal dimensions fields of the aforementioned series and their deviations from average on three frames (kernels) with size: $2 \times 2,4 \times 3,8 \times 6$ (width $\times$ height). The procedure was the following: we partitioned thermograms accordingly to the frames sizes, determined the temperature mean values in every part of thermal image, and then ascertained the absolute deviations from corresponding mean for each pixel in the specified frame. After that, we generated grayscale and RGB images and used ImageJ software packages to build fractal dimension field. According to the definitions Eq. (1) and (2), the arithmetic average $\bar{T}_{k l}$ and deviation $d_{k l}$ with kernel size $2 \times 2,4 \times 3,8 \times 6$ pixels were obtained. It necessary to notice, the searching masks for each kernel were developed to optimize the processing of data lists (76800 elements) and reduced arrays (76800/kernel size), that greatly increased time effectiveness.

$$
\begin{gathered}
\bar{T}_{k l}=\frac{1}{n m} \sum_{i, j}^{i=i+n, j=j+m} T_{i j} \\
d_{i j}=\sqrt{\frac{1}{n m} \sum_{i, j}^{i=i+n, j=j+m}\left(T_{i j}-\bar{T}_{k l}\right)^{2}}
\end{gathered}
$$

\section{Main results}

Conformably to the technical-water supply control system on NPP, that is used to cool the turbine condensers, it is possible to distinguish several groups of objects subjected to state estimation: buried pipelines, stop valves, pumping facility, facilities of spray ponds. The surface temperature was successfully determined under the control mode of valves. We succeeded also in finding of several painted coating peeling in initial stage. Investigations were conducted at ambient temperature $25^{\circ} \mathrm{C}$ and $2^{\circ} \mathrm{C}$, the approximate temperature of water was $15^{\circ} \mathrm{C}$. Obviously, the defects detection was more effective at lower temperature. Thermograms of spray ponds shields and pumping facility were made (figure 1 ).
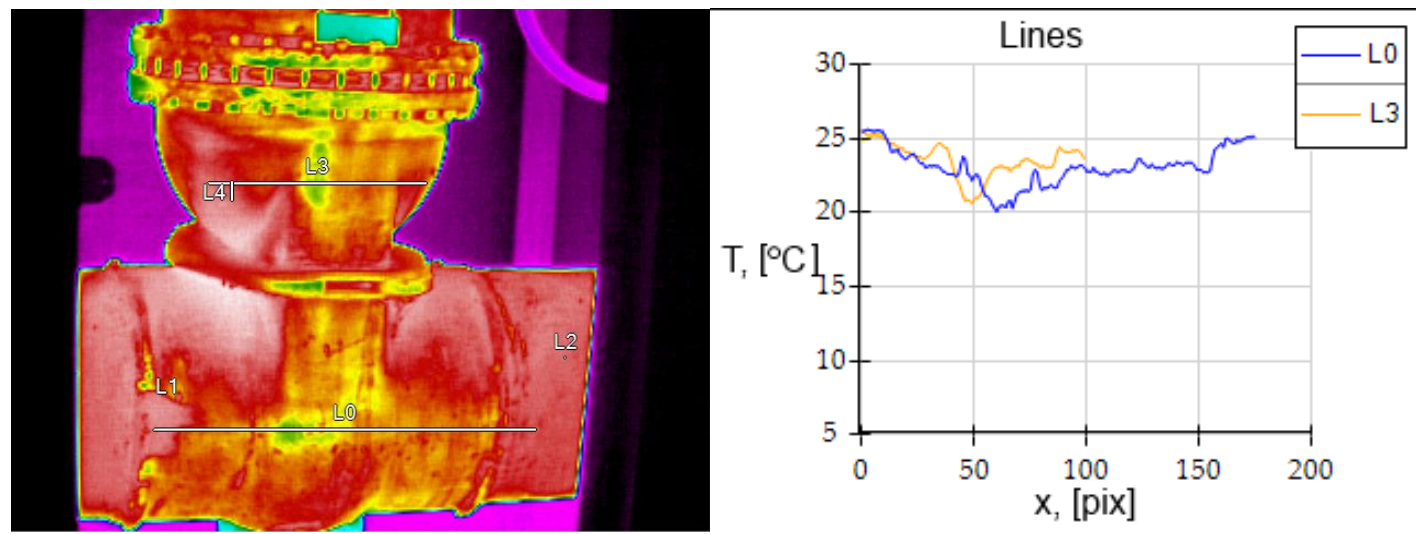

Fig. 1. Valve thermogram and corresponding temperature profile on its surface

The most complex feature is the integrity testing of buried pipelines because of inflation pressures at is not enough for using of ultrasonic leak testers, especially for low pressure pipelines which carries water form spray bonds.

At the same time, in the case of leakage formation, soil moisture can be expected and the heat occurrence on surface can be observed consequently by thermal techniques as for instance in [9]. In addition, the situation is complicated by the pipelines that are buried, placed on water level. In this case, the heat occurs by the evolutionary way: soil washout, shift of moisture transfer regime, which can become apparent as changes of surface microrelief. Such heterogeneity of coating as concrete slabs, crushed stone, asphalt and greenery make our problem more complicated. Meanwhile, extensive temperature gradients weren't observed within the bounds of coating homogeneity.

The work next idea was to apply fractal analysis to enhance informativeness of thermograms. In general, fractal dimension of thermal image depends on its visualization type. If we consider a thermogram as a binary image, its fractal dimensions ranges from 1 to 2, it has two possible values each pixel. At second, a grayscale image is a 3-dimensions object, each pixel $(x, y)$ has luminance $z=f(x, y)$ with 256 possible values. In this case a thermography is a surface, and the fractal dimensions interval is $\{2,3\}$. A color thermogram is a hyper-surface in a color space. For example, if we deal with RGB image, then we handle with ( $x, y, R, G, B)$ 5-D vectors in a 5-D Euclidian hyper-space [3,6].

In this work we establish the multifractal dimensions $D_{M F}$ and local connected fractal dimensions $D_{\text {LCFD. We can }}$ use this information to characterise the heat sources. We can localize these heat sources, or predict the heat flow from the object. 
Table 1. Thermograms of the soil surface with grass cover (taken at $t=0,+15,+30 \mathrm{~min}$ ) with the corresponding distributions of deviations. Deviations are filtered in preference to abmodality $\Delta=\left|T_{i j}-\bar{T}_{k l}\right|>0.05 K$.

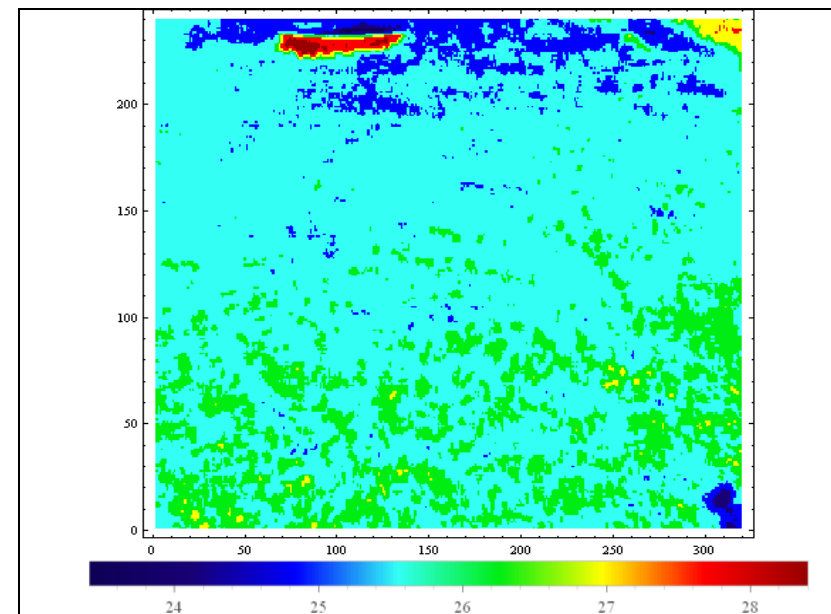

Fig. 2. Thermogram IR110, $t=0 \mathrm{~min}$, $D_{M F}=2.79 . .2 .82$

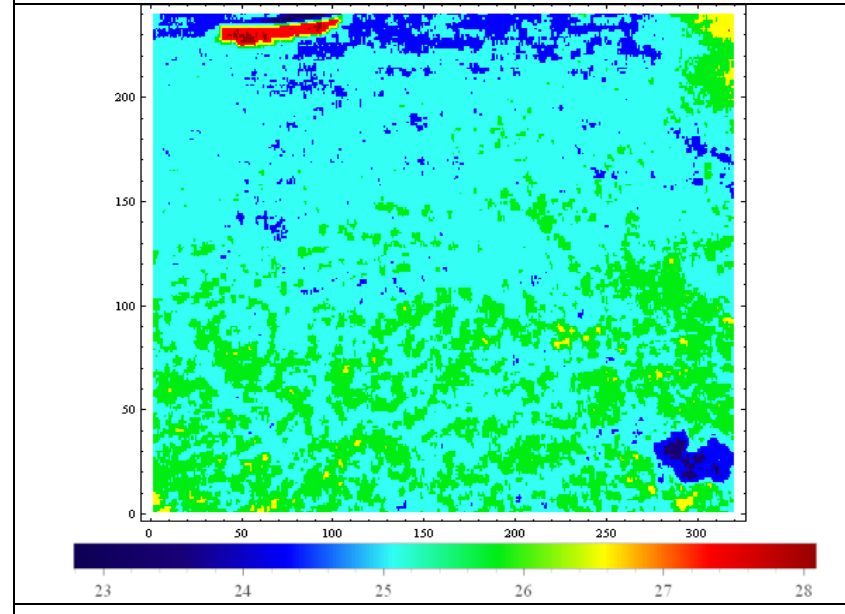

Fig. 4. Thermogram $I R 111, t=15 \mathrm{~min}$, $D_{M F}=2.75 . .2 .82$

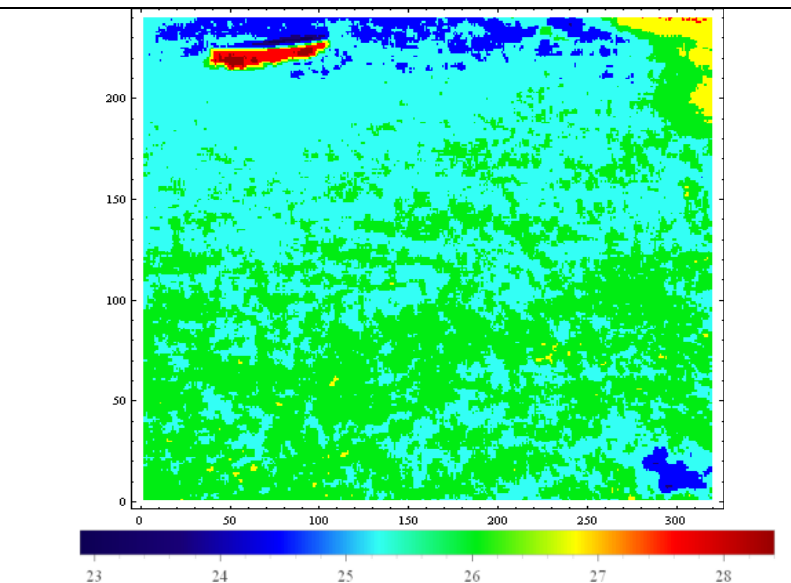

Fig. 6. Thermogram IR112, $t=30 \mathrm{~min}$, $D_{M F}=2.78 . .2 .86$

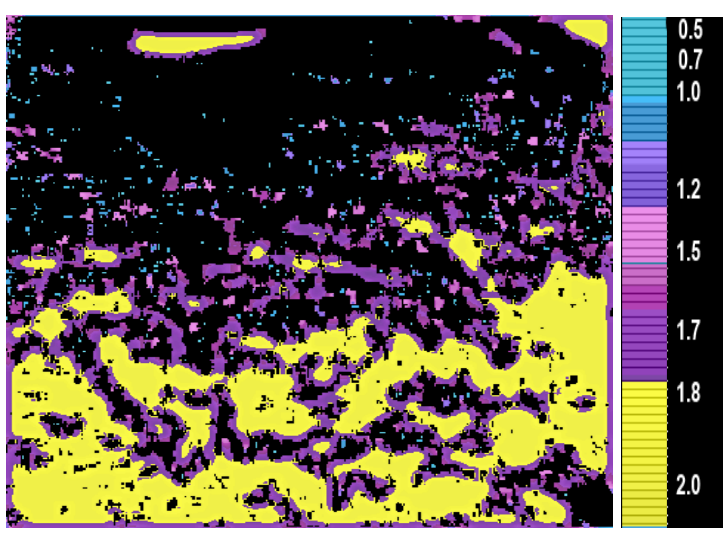

Fig. 3. Local connected fractal dimension map of IR110, $t=0 \mathrm{~min}$,

$D_{L C F D}=1.0 . .1 .8$. Black color - background.

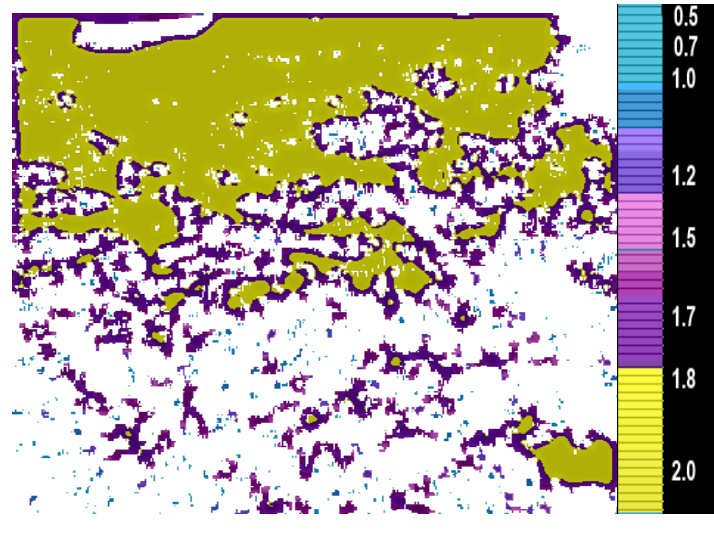

Fig. 5. Local connected fractal dimension map of IR111, $t=15 \mathrm{~min}$,

$D_{L C F D}=1 . .1 .9$. White color - background.

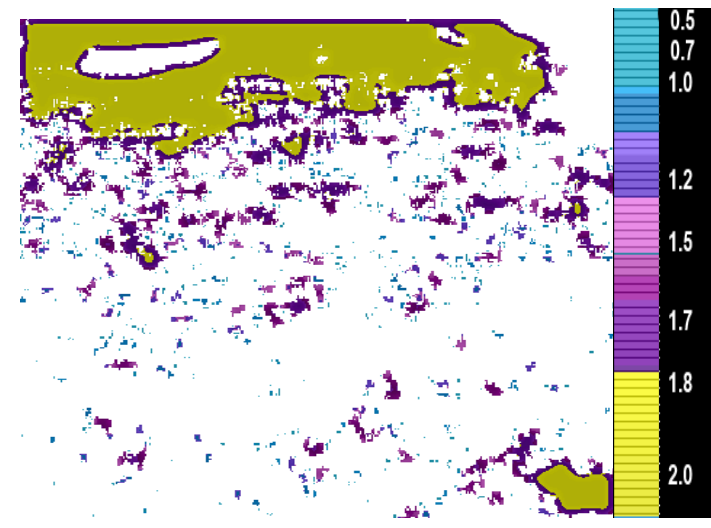

Fig. 7. Local connected fractal dimension map of IR112, $t=30 \mathrm{~min}$,

$D_{L C F D}=1$..1.9. White color - background. 
We conducted the multifractal analysis and local connected fractal dimension analysis of the sequence of three thermograms $T_{i j}$ of the soil surface with grass cover (table 1) and of the raster images of their deviations $d_{k l}$ (table 2) from the local average. Fractal characteristics of $d_{k l}$ distributions were calculated for low and high abmodalities. The results of calculations are presented in table 3. It was shown that the fractal dimensions of these thermograms $D_{M F} \sim 2.8$ are lower than their deviations $\sim 2.87$ (figure 10). The spread of the dimensions intervals $(\sim 0.1)$ is caused by the disadvantages of the oblique, tilted away from vertical measurements, low matrix resolution and the surface heterogeneity.

Table 2. Thermograms of the soil surface with grass cover (taken at $t=0,+15,+30 \mathrm{~min}$ ) with the corresponding distributions of deviations. Deviations are filtered in preference to abmodality $\Delta=\left|T_{i j}-\bar{T}_{k l}\right|>0.05 K$.

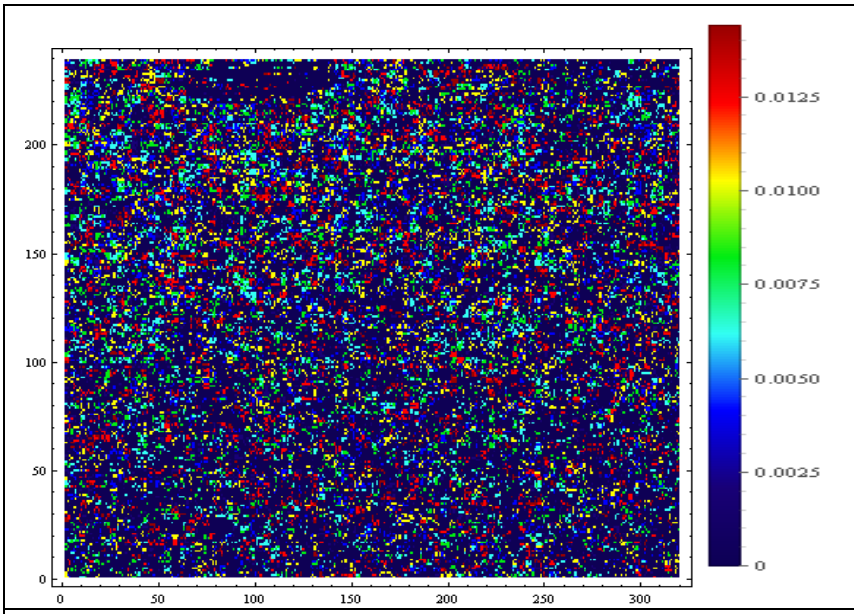

Fig. 8. IR 110, deviation distribution, kernel size $4 \times 3$, filtered by $\Delta>0.05 \mathrm{~K}, \mathrm{t}=0 \mathrm{~min}, \mathrm{D}_{\mathrm{MF}}=2.82 . .2 .92$

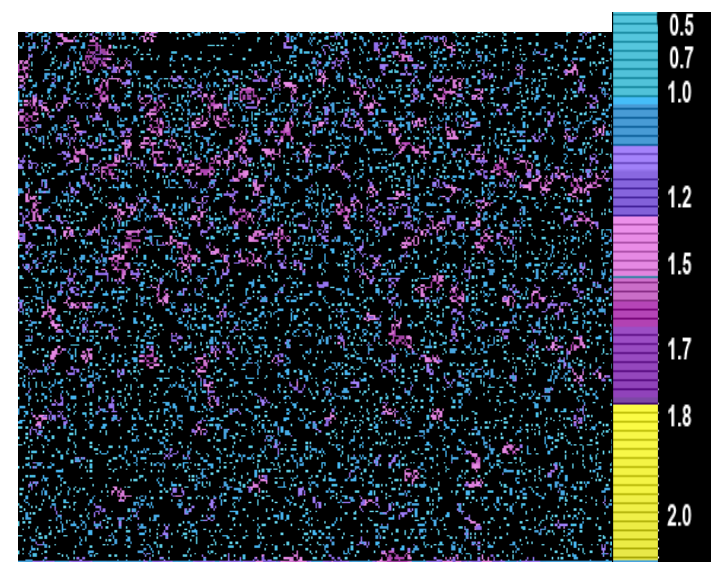

Fig. 9. IR 110, local connected fractal dimension map for deviation distribution, kernel size $4 \times 3$, filtered by $\Delta>0.05 \mathrm{~K}$, $\mathrm{t}=0 \mathrm{~min}, \mathrm{D}_{\mathrm{LCFD}}=0.7 . .1 .7$

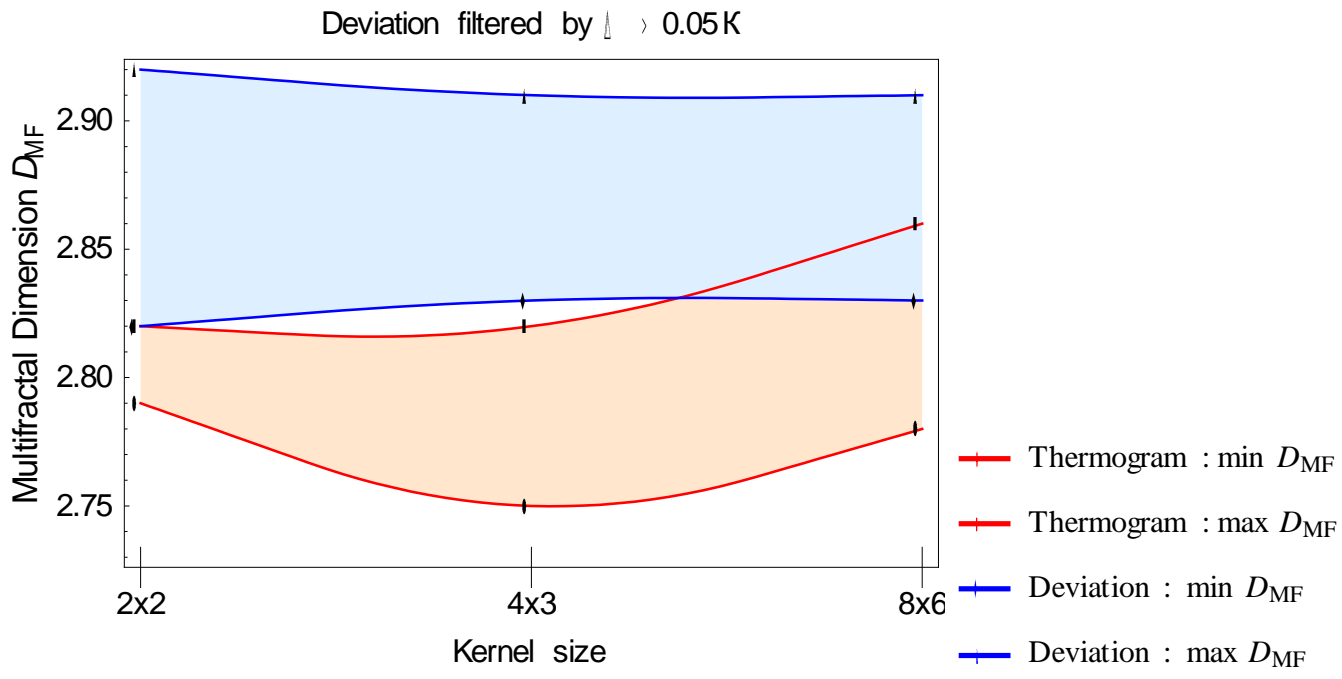

Fig. 10. Multifractal dimension intervals $D_{M F}$ for three thermograms (red) and their deviations (blue).

After reduction of thermograms' resolution (table 3, figures 11 -13) through averaging over the frame (kernel), both the temperature distributions and the multifractal dimension intervals were significantly changed. The dependence of reduction of the thermogram of the soil surface with grass cover (IR 110) on the kernel size $2 \times 2,4 \times 3,8 \times 6$ (width $x$ height) was shown on figure 14. The corresponding fractal dimension intervals decrease almost linearly with reduction of image resolution, ie, with increase of the averaging kernel size. 
Table 3. Reduced images of thermogram IR 110 for different kernel sizes.

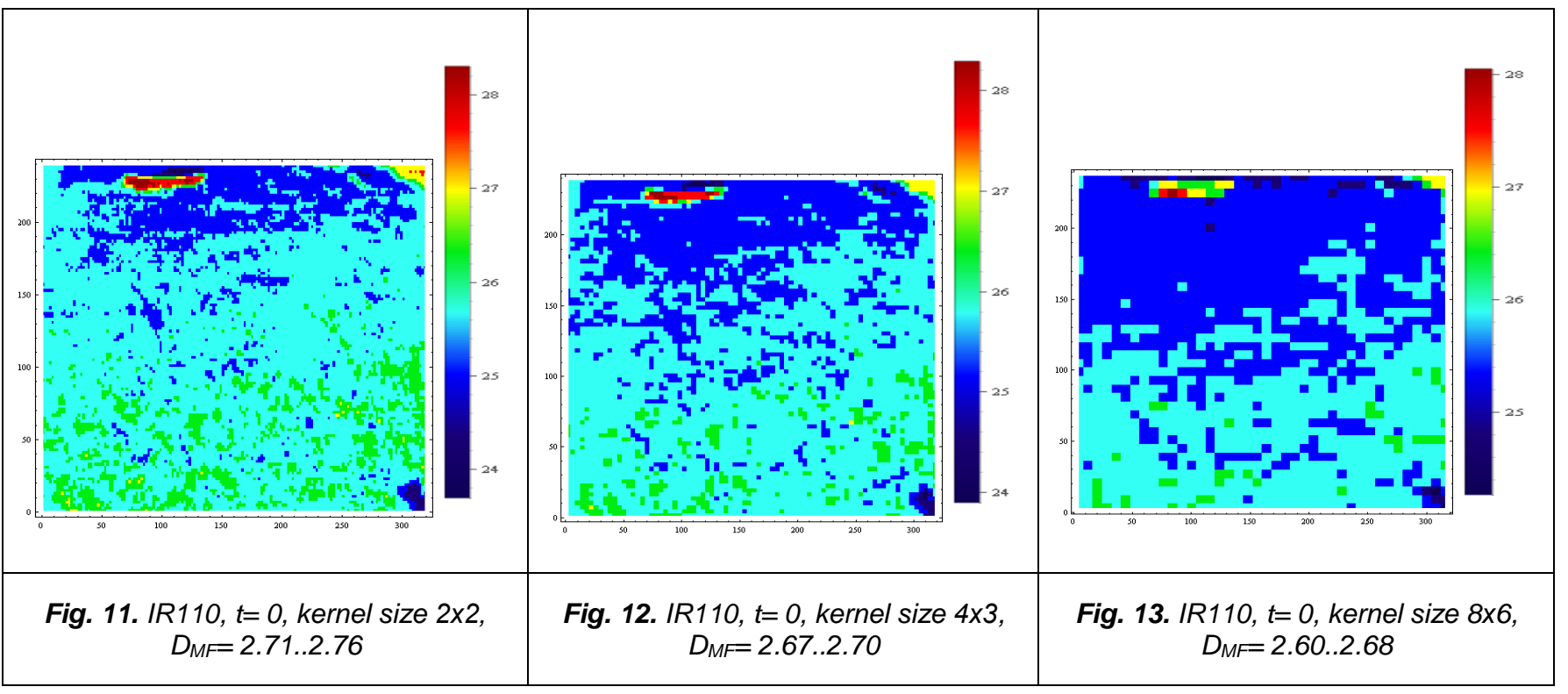

The dependence of fractal scaling characteristics on the kernel size for filtered deviations was also obtained. The imposed conditions on deviation were: we take into account only those pixels which abmodality in kernel is only less or only more than the absolute accuracy at measuring temperature $\sim 0.05 \mathrm{~K}$. It was shown (figure 15, 16), that the aforecited filter condition changed the dependence to the opposite: if the filter condition is $\Delta=\left|T_{i j}-\bar{T}_{k l}\right| \leq 0.05 \mathrm{~K}$ then the multifractal dimension decreases when the kernel size increases, if the filter condition is $\Delta=\left|T_{i j}-\bar{T}_{k l}\right|>0.05 \mathrm{~K}-$ otherwise. Such behaviour of $D_{M F}$ are explained by the segmentation of data - if $\Delta \leq 0.05 \mathrm{~K}$ we investigate small local changes in temperature, while $\Delta>0.05 \mathrm{~K}$ - we consider the extremal differences of thermogram.

Table 4. The dependence of the fractal dimension $D_{M F}$ of thermograms and their deviations on kernel size. Deviations are filtered accordingly to abmodality $\Delta>0.05 K$ or $\Delta \leq 0.05 K$.

\begin{tabular}{|c|c|c|c|c|c|c|c|c|c|}
\hline \multirow{4}{*}{$\begin{array}{l}\text { Ker } \\
\text { nel }\end{array}$} & \multicolumn{9}{|c|}{$\mathrm{D}_{\mathrm{MF}}$} \\
\hline & \multicolumn{3}{|c|}{ IR $110, t=0 \min$} & \multicolumn{3}{|c|}{ IR $111, t=15 \min$} & \multicolumn{3}{|c|}{ IR $112, t=30 \mathrm{~min}$} \\
\hline & \multirow[t]{2}{*}{ thermogram } & \multicolumn{2}{|c|}{ deviation } & \multirow[t]{2}{*}{ thermogram } & \multicolumn{2}{|c|}{ deviation } & \multirow[t]{2}{*}{ thermogram } & \multicolumn{2}{|c|}{ deviation } \\
\hline & & $\begin{array}{l}\Delta \leq \\
0.5 K\end{array}$ & $\begin{array}{l}\Delta> \\
0.05 K\end{array}$ & & $\begin{array}{l}\Delta \leq \\
0.05 K\end{array}$ & $\begin{array}{l}\Delta> \\
0.05 K\end{array}$ & & $\begin{array}{l}\Delta \leq \\
0.05 K\end{array}$ & $\begin{array}{l}\Delta> \\
0.05 K\end{array}$ \\
\hline $2 \times 2$ & $2.71 . .2 .76$ & $\begin{array}{l}2.91 . . \\
3.00\end{array}$ & $\begin{array}{l}2.63 . . \\
2.67\end{array}$ & $2.71 . .2 .80$ & $\begin{array}{l}2.91 . . \\
2.98\end{array}$ & $\begin{array}{l}2.57 . . \\
2.65\end{array}$ & $2.72 . .2 .78$ & $\begin{array}{l}2.91 . . \\
3.00\end{array}$ & $\begin{array}{l}2.70 . . \\
2.74\end{array}$ \\
\hline $4 \times 3$ & $2.67 . .2 .70$ & $\begin{array}{l}2.82 . . \\
2.92 \\
\end{array}$ & $\begin{array}{l}2.79 . . \\
2.85 \\
\end{array}$ & $2.65 . .2 .71$ & $\begin{array}{l}2.83 . . \\
2.91 \\
\end{array}$ & $\begin{array}{l}2.79 . . \\
2.84 \\
\end{array}$ & $2.67 . .2 .74$ & $\begin{array}{l}2.83 . . \\
2.91 \\
\end{array}$ & $\begin{array}{l}2.80 . . \\
2.85 \\
\end{array}$ \\
\hline $8 \times 6$ & $2.60 . .2 .68$ & $\begin{array}{l}2.79 . . \\
2.83\end{array}$ & $\begin{array}{l}2.73 . . \\
2.79\end{array}$ & $2.58 . .2 .68$ & $\begin{array}{l}2.79 . . \\
2.85\end{array}$ & $\begin{array}{l}2.71 . . \\
2.80\end{array}$ & $2.56 . .2 .65$ & $\begin{array}{l}2.79 . . \\
2.86\end{array}$ & $\begin{array}{l}2.76 . . \\
2.82\end{array}$ \\
\hline
\end{tabular}

Finally, the obtained experimental thermograms are noisy, so it was necessary to study how random noise influences on the thermograms. A random noise with amplitude $0.05 \mathrm{~K}$ was added directly to the thermogram IR 110 , but significant changes in the fractal scaling were not observed. After that, we increased the noise amplitude up to $0.25 \mathrm{~K}$, and only $5 \%$ narrowing of the dimension intervals was occurred. So, we can conclude, that the fractal dimension distributions are stable to slightly perturbation noise. 


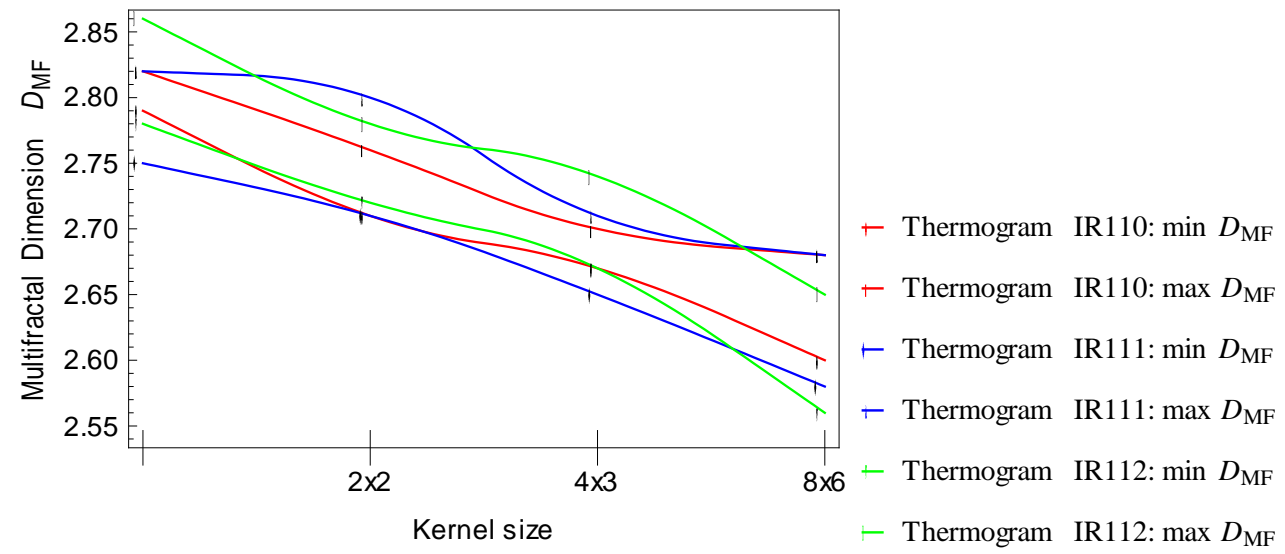

Fig. 14. The dependence of the multifractal dimension intervals $D_{M F}$ on kernel size (width $x$ height) for thermograms IR 110, IR 111, IR 112.

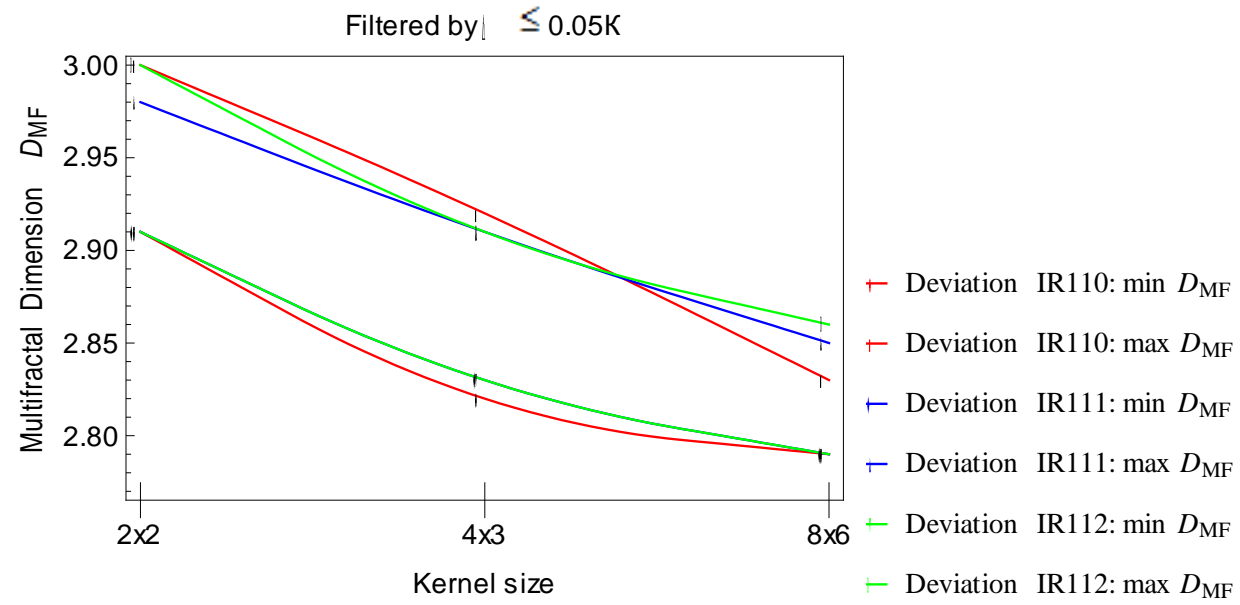

Fig. 15. The dependence of the multifractal dimension intervals $D_{M F}$ on kernel size (width $x$ height) for filtered deviations (IR 110, IR 111, IR 112). Filter condition: $\Delta=\left|T_{i j}-\bar{T}_{k l}\right| \leq 0.05 K$.

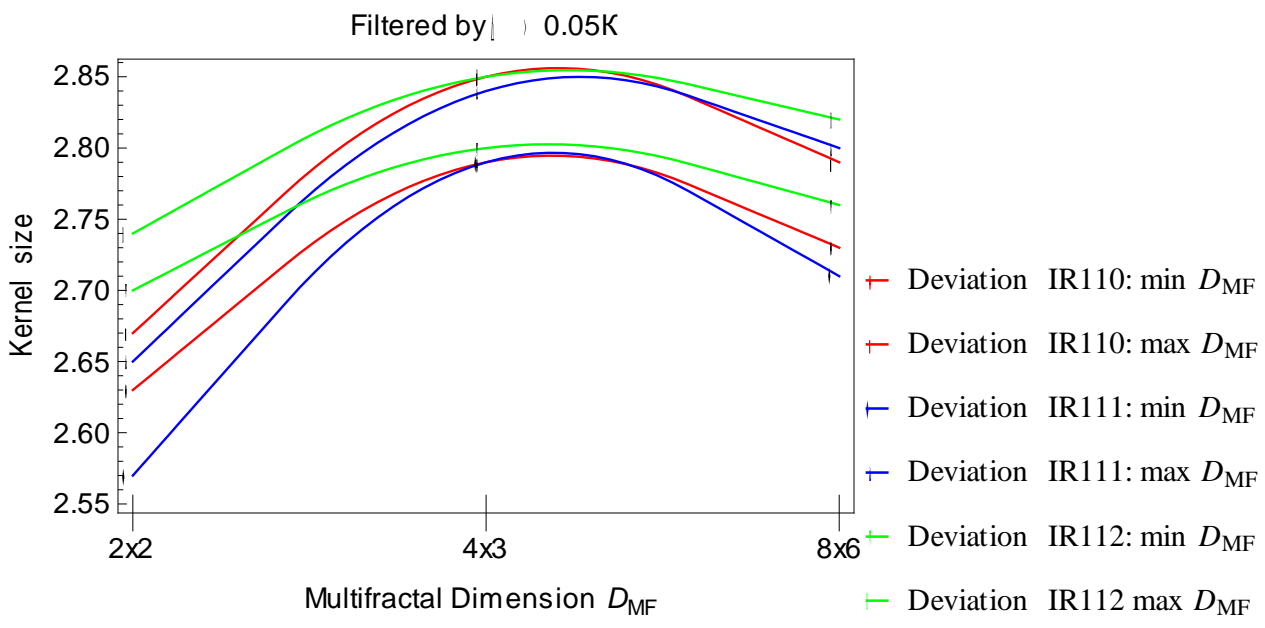

Fig. 16. The dependence of the multifractal dimension intervals $D_{M F}$ on kernel size (width $x$ height) for filtered deviations (IR 110, IR 111, IR 112). Filter condition: $\Delta=\left|T_{i j}-\bar{T}_{k l}\right|>0.05 \mathrm{~K}$ 


\section{Conclusion}

It is reasonable to set the stationary points of observation and analyze dynamics of parameters that characterized temperature field to provide early diagnostics of the developed leakage, to detect temperature anomalies in the equipment, especially, underground facilities.

As a complementary method for thermogram processing it is possibly to use fractal analyses. We demonstrated the generally usefulness of the method of fractal analysis in IR thermography monitoring. A better understanding of the fractal character of thermograms will enable engineers to provide guidance in determining of heat peculiarities. Quantitative characterization of fractal complexity of thermographic data complements conventional statistical analysis techniques and provides additional insight into the nature of the problem.

\section{REFERENCES}

[1] David Cornette. "Building a Marble Pattern Using Fractal Noise", http://www.davidcornette.com/glsl/noise.html

[2] Манфред Шредер. “Фракталы, хаос, степенные законы”, Ижевск, с. 528, 2001.

[3] N.Selvarasu, Alamelu Nachiappan, N.M.Nandhitha. "Euclidean Distance Based Color Image Segmentation of Abnormality Detection from Pseudo Color Thermographs", International Journal of Computer Theory and Engineering, Vol. 2, No. 4, 1793-8201, 2010.

[4] Daniel Shiffman. "The Nature of Code", Magic Book Project, p. 468, 2012.

[5] Edrees M. N. Mahmood. "Applications of Fractal Dimension”, Iraqi Journal of Statistical Science (10), pp. 54-73, 2006.

[6] Mihai Ivanovici, Noël Richard. "Fractal Dimension of Color Fractal Images", IEEE Transactions on Image Processing, Vol. 20, No. 1, pp. 227-235, 2011.

[7] http://imagej.nih.gov/ij/plugins/fraclac/FLHelp/Glossary.htm\#db

[8] Mark A. Johnson, Lawrence V. Meisel. "Scaling Analysis of Thermographic Images Using Neural Networks", Technical Report Arccb-Tr-97010, Us Army Armament Research, p. 15, 1997.

[9] Mohamed Fahmy, Osama Mjselhi." Detecting and locating leaks in underground water mains using thermography", Proceedings of 26th International Symposium on Automation and Robotics in Construction (ISARC 2009), pp. 61-67, 2009. 